\title{
Parámetros genéticos de las características andrológicas en la especie bovina
}

\author{
Genetic parameters of andrological traits in bovine \\ MR Silva $^{a}$, VB Pedrosa ${ }^{b}$, JBC Silva $^{c^{*}}$, LGG Herrera ${ }^{d}$, JP Eler $^{b}$, LG Albuquerque $^{a}$ \\ aDepartamento de Zootecnia, Universidad Estadual Paulista, FCAV-UNESP, Jaboticabal, São Paulo, Brasil.

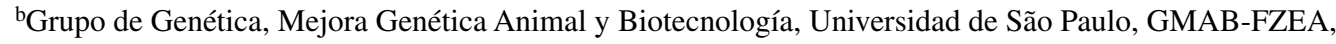 \\ Pirassununga, São Paulo, Brasil. \\ 'Departamento de Medicina Preventiva Veterinaria y Reproducción Animal, Universidad Estadual Paulista, FCAV-UNESP, \\ Jaboticabal, São Paulo, Brasil. \\ ${ }^{\mathrm{d} F a c u l t a d}$ de Ciencias Agropecuarias, Universidad Nacional de Colombia, Medellín, Colombia.
}

\begin{abstract}
SUMMARY
The sexual precocity and fertility of bovines have great impact on the economic success of commercial cattle herds, where some reproductive traits have been adopted as selection criteria. However, the majority of these traits depend on reproductive events of the females and, with exception of the scrotal circumference, few studies approach others andrological traits. The estimation of heritabilities and genetic correlations of testicular and seminal traits and also sexual behaviour, will allow to provide alternatives for the design of more appropriate selection strategies for fertility, together with other economic traits.
\end{abstract}

Palabras clave: bovinos, correlación genética, examen andrológico, heredabilidad.

Key words: cattle, genetic correlation, breeding soundness, heritability.

\section{INTRODUCCIÓN}

El impacto del aumento de la fertilidad y/o uso de animales genéticamente seleccionados para características de interés económico son determinantes en el progreso de la productividad en rebaños comerciales. En Brasil, por ejemplo, se estima que 45,2\% (Anualpec 2010) de los 205,3 millones de bovinos (IBGE 2010) está compuesto por hembras en edad reproductiva, la mayoría de ellas sometidas a sistema de monta natural, dado que la inseminación artificial se encuentra restringida a menos del $8 \%$ de estas hembras. En estas condiciones, las pérdidas en la productividad podrían ser expresivamente minimizadas por la eliminación de toros con baja capacidad reproductiva y, principalmente, por la utilización de toros con evaluación genética deseable para las características económicamente importantes, como la fertilidad.

Algunas características reproductivas son utilizadas como criterios de selección en búsqueda del incremento de la precocidad sexual y de la fertilidad, a pesar de sufrir gran influencia del ambiente y de presentar baja heredabilidad. La inclusión y el estudio de esas características en distintos programas de mejoramiento genético son de

Aceptado: 04.08.2011.

* Vía de acceso Prof. Paulo Donato Castellane s/n, 14884-900, Jaboticabal-SP, Brasil; julicborges@yahoo.com.br gran importancia, principalmente de registros de eventos reproductivos de las hembras, entre los cuales se destacan la edad al primer parto, facilidad de partos, intervalo de partos, número de días para el parto, período de gestación, longevidad reproductiva o "stayability" y la probabilidad de preñez temprana.

En los machos, solamente el perímetro escrotal (PE) es adoptado como criterio de selección para aumento de la fertilidad en los programas de mejoramiento genético de bovinos. La principal importancia dada al perímetro escrotal se refiere a la alta magnitud de la heredabilidad y su correlación genética favorable con la precocidad sexual y longevidad reproductiva de las hembras emparentadas.

Además del PE, otras características provenientes de informaciones fisiopatológicas que integran el examen andrológico podrán ser relevantes en estudios genéticocuantitativos. Sin embargo, pocos estudios fueron realizados con el objetivo de estimar parámetros genéticos de las características testiculares, seminales y de comportamiento sexual provenientes de los exámenes andrológicos que podrían promover direccionamientos más adecuados de la selección para fertilidad en rebaños comerciales, especialmente en sistemas de producción con utilización de monta natural. Esta revisión pretende describir algunos aspectos importantes sobre la obtención de parámetros genéticos de las características andrológicas en búsqueda de nuevos criterios de selección para el aumento de la fertilidad en rebaños bovinos. 


\section{ESTUDIOS GENÉTICOS CUANTITATIVOS CON CARACTERÍSTICAS ANDROLÓGICAS EN BOVINOS}

La comprensión de la importancia del examen andrológico como predictor de la aptitud reproductiva y de la obtención de parámetros genéticos fundamenta los estudios genéticos en la búsqueda de nuevos criterios de selección de toros. El conocimiento del perfil cuantitativo y cualitativo de la producción seminal de reproductores, dotados de alta fertilidad y superiores genéticamente, es importante en el incremento de la fertilidad general del rebaño (Rege y col 2000).

El examen andrológico, utilizado como medida de la capacidad o aptitud reproductiva de los toros (Fonseca y col 1997, Kealey y col 2006), tiene como objetivo la evaluación clínica de los órganos genitales y de los aspectos clínicos generales, además del espermograma, que consiste en el examen físico del eyaculado y de la morfología de las células espermáticas. Alexander (2008), en referencia a las normas adoptadas por la Sociedad Americana de Theriogenología en 1993 (Chenoweth y col 1993), destaca que esta evaluación requiere el cumplimiento de patrones mínimos de cuatro categorías, examen físico general y reproductivo, medida del PE de acuerdo con la edad, así como la evaluación de la motilidad y morfología del semen. En Brasil, los estándares seminales deseables para efectos de selección de toros para monta natural fueron establecidos por el Colegio Brasileño de Reproducción Animal (Henry y Neves 1998). De acuerdo con estos criterios, para que el reproductor sea considerado apto debe poseer los estándares mínimos de $70 \%$ de motilidad espermática progresiva rectilínea (0-100\%), calificación 3 del vigor espermático (0-5), calificación 3 de turbidez o movimiento en masa (0-5) y como máximo $30 \%$ del total de espermatozoides anormales. El PE también es determinante en la clasificación de toros jóvenes sometidos a la evaluación andrológica y diferentes estándares de medidas mínimas son establecidas en conformidad con la edad y subespecie animal (Bos taurus y Bos indicus) (Henry y Neves 1998). Por consiguiente, la evaluación andrológica dispone de una serie de informaciones de naturaleza numérica o categórica que almacenadas en bancos de datos específicos podrán ser estudiadas para la obtención de parámetros genéticos.

Las estimativas de parámetros genéticos, o sea, de la heredabilidad y de las correlaciones genéticas, son esenciales para la selección artificial, con base en los efectos genéticos aditivos, para una determinada característica de interés. En estudios de características cuantitativas, la principal función de la heredabilidad es su carácter predictivo que expresa la confiabilidad del valor fenotípico como indicador del valor genético, o sea, mide el grado de correspondencia entre el fenotipo y el valor genético (Euclides Filho 1999), mientras que la correlación genética aditiva, cuya causa principal es la pleiotropía, indica el grado en que dos caracteres están influenciados por los mismos genes, o por genes estrechamente ligados que determinan caracteres diferentes (Falconer y Mackay 1996). Sin embargo, en el contexto de selección la correlación genética es importante por indicar el cambio de un carácter en función de otro, lo que permite hacer predicciones al respecto e incluso enfocar los trabajos de selección para una determinada característica buscando respuesta en otra genéticamente relacionada, lo que es conocido como selección indirecta (Falconer y Mackay 1996).

La demanda del examen andrológico en toros jóvenes oriundos de programas de mejoramiento genético es ascendente, una vez que esta evaluación es determinante en la aptitud reproductiva y también como estrategia de marketing para la comercialización (Kealey y col 2006). Gracias a ello, los bancos de datos tienden a ser más robustos en cantidad y calidad de las medidas andrológicas de toros con ascendencia conocida, posibilitando los análisis en el área de la genética cuantitativa. Por esta razón, la importancia de estimar la heredabilidad y las asociaciones genéticas, fenotípicas y ambientales entre las características andrológicas y de estas con otras de interés económico fue considerada por algunos investigadores (Smith y col 1989, Dias y col 2006, Folhadella y col 2006, Kealey y col 2006).

La disponibilidad de publicaciones científicas sobre parámetros genéticos de características andrológicas es poca, posiblemente debido a limitaciones de orden práctica, como son: la necesidad de conocimiento y capacitación técnica para la realización y estandarización de los exámenes andrológicos (Silva 2009), y la indisponibilidad de bancos de datos compuestos por registros de animales con parentesco conocido. A esto se le suma el hecho de que algunas de las características andrológicas son de naturaleza categórica y muchas veces dotadas de evaluaciones subjetivas cuando consideras lineares en las evaluaciones genéticas (como, por ejemplo, motilidad y vigor espermático), exigiendo estandarización en la determinación de las calificaciones y adecuación en los procedimientos estadísticos y computacionales para la estimación de los parámetros genéticos.

Entre las publicaciones científicas en el área de genética cuantitativa, las características andrológicas de bovinos están dentro de las más difundidas. Algunos estudios genéticos también han sido realizados en otras especies domésticas, como en aves (Kabir y col 2007), cerdos (Oh y col 2003, Robinson y Buhr 2005, Smital y col 2005, Oh y col 2006, Wolf 2009, Wolf 2010), ovinos (Rege y col 2000, Snowder y col 2004, David y col 2007), caprinos (Furstoss y col 2009), conejos (Brun y col 2002, García-Tomáz y col 2006, Lavara y col $2008^{\mathrm{a}, \mathrm{b}}$ ), equinos (Van Eldik y col 2006) y perros (England y col 2010), especialmente incluyendo características seminales. Probablemente el mayor interés científico en estudios con especies de producción sea reflejo de la preocupación de diferentes grupos de investigación en buscar nuevos criterios de selección para el aumento de la fertilidad con base en las características andrológicas, debido al 
destacado impacto económico del aumento de la fertilidad en ganaderías comerciales.

Dentro de los primeros reportes de estimaciones de parámetros genéticos de características andrológicas en bovinos, se destacaron los trabajos pioneros de Abadia y col (1976) y Coulter y col (1976), seguidos por Schlote y Munks (1980), que las asociaron a datos de fertilidad en campo. Otros estudios con toros donadores de semen de razas europeas fueron publicados en secuencia, con estimaciones de heredabilidad de moderadas a altas magnitudes para las características de producción espermática (Knights y col 1984, Chandler y col 1985, Taylor y col 1985, Graffer y col 1988, Stalhåmmar y col 1989, Ducrocq y Humblot 1995, Ducrocq y Humblot 1998). Estos estudios contribuyeron para la discusión crítica de la inclusión de características testiculares, seminales y de comportamiento sexual del macho como criterios de selección alternativos al PE, dada la importancia de estas en la determinación clínica de la fertilidad del toro.

En esta búsqueda de nuevos criterios de selección considerando las características andrológicas, Quirino (1999) reportó estimaciones de heredabilidad y correlaciones genéticas entre medidas testiculares, características seminales y libido de datos provenientes de 438 toros de la raza Nelore (Bos indicus) criados extensivamente en Brasil. Dado que cerca del $80 \%$ del rebaño bovino brasileño está compuesto por animales de razas cebuinas o fruto del cruzamiento con ellas, sobre todo de la raza Nelore, y que la monta natural es preponderante, el referido trabajo científico fue relevante por contribuir en el abordaje de las características andrológicas buscando la selección de toros cebuinos criados bajo condiciones extensivas. A pesar de que el número de animales incluidos puede ser considerado bajo para análisis genéticos, Quirino (1999) fue pionera en obtener parámetros genéticos de la libido y por indicar el PE como característica indicadora de la calidad espermática en toros Nelore con base en las correlaciones genéticas favorables obtenidas.

Estudios posteriores con características andrológicas en Bos indicus en Brasil (Martinez y col 2000, Sarreiro y col 2002, Quirino y col 2004, Silveira 2004, Dias y col 2006, Silva 2009, Silva y col 2011) y con Bos taurus en países del hemisferio norte (Kealey y col 2006, Garmyn y col 2011) incluyeron diferentes metodologías de análisis genéticos con el objetivo de mejorar la calidad de la estimación de los parámetros genéticos, aumentar el número de características analizadas simultáneamente (análisis multicaracterísticos), minimizar los errores estándar y maximizar la precisión de predicción de los valores genéticos de los animales para poder hacer inferencias más seguras.

Utilizando registros de 807 colectas de semen de 105 toros de la raza Gir seleccionados para leche de diferentes centrales de inseminación artificial, Martinez y col (2000) estimaron heredabilidad y correlaciones genéticas para PE, concentración espermática, aspectos físicos (motilidad, vigor y turbidez) y defectos espermáticos. Con base en las correlaciones obtenidas, en general en sentido favorable, los autores sugirieron que el PE puede ser utilizado como característica de eliminación previa de los toros que son candidatos a la colecta de semen.

Sarreiro y col (2002) y Quirino y col (2004) reportaron parámetros genéticos de la libido, además de características seminales, PE y peso corporal en 273 y 288 toros de la raza Nelore, respectivamente. Los autores indicaron que la respuesta a la selección directa para PE y libido así como la selección para una de ellas traería respuesta correlacionada entre las dos características de manera favorable. En esta misma raza, Dias y col (2006) estimaron la heredabilidad y las correlaciones genéticas entre características ponderales, de biometría, consistencia testicular y espermograma en 579 toros jóvenes. En este estudio también fue contemplado el índice de clasificación andrológica por puntos (CAP) (Vale Filho 1989, Vale Filho y col 1999). Las estimaciones de heredabilidad fueron bajas para las características seminales y CAP, siendo consideradas altas apenas para PE y consistencia testicular, concordando con estudios previos que determinaron el pequeño componente genético aditivo para las características seminales. Frente a estos resultados de las correlaciones genéticas entre las características estudiadas, los autores sugirieron que hay compatibilidad entre crecimiento corporal y fertilidad en los programas de selección de reproductores Nelore.

La precisión de las estimaciones de parámetros genéticos es dependiente del número de datos analizados, y uno de los primeros estudios con registros andrológicos de 5.903 toros Nelore fue realizado por Silveira (2004). Posteriormente, Fernandes Júnior (2006), con datos de 3.636 toros jóvenes compuestos Montana Tropical de aptitud para carne (Ferraz y col 1999, Fernandes y Franceschini 2007), estimó también parámetros genéticos para $\mathrm{PE}$, volumen testicular y aptitud reproductiva, con altas magnitudes de heredabilidad para las características testiculares.

Silva y col (2011) estimaron heredabilidad y correlaciones genéticas para estas características con más de 17.600 toros jóvenes de la raza Nelore, haciendo uso de técnicas estadísticas de inferencia bayesiana intentando minimizar los errores estándares inherentes a los análisis; las estimativas de heredabilidad presentaron magnitudes de moderadas a altas para características testiculares y bajas para la aptitud andrológica y características seminales, confirmando los resultados anteriores. Las correlaciones genéticas, en este estudio, se mostraron favorables entre las características testiculares y seminales, resaltando que la selección para el perímetro y volumen testicular deberá promover rápido progreso genético y contribuir para la mejoría de la calidad del semen de toros jóvenes. Corroborando estos hallazgos, en Bos taurus, Garmyn y col (2010) encontraron correlaciones genéticas favorables y de baja magnitud entre PE y características seminales en 1.281 toros de la raza Angus.

De modo general, los artículos sobre estimaciones de parámetros genéticos de las características andrológicas 
fueron resumidos en características testiculares resultantes del examen clínico de los testículos y bolsa escrotal, características seminales obtenidas en espermograma y la libido observada en esta prueba específica de comportamiento sexual.

\section{PARÁMETROS GENÉTICOS DE LAS CARACTERÍSTICAS TESTICULARES}

\section{PERÍMETRO ESCROTAL (PE)}

El perímetro escrotal es la característica reproductiva más utilizada en programas de mejoramiento genético de bovinos y, al contrario de las demás características andrológicas, es ampliamente abordada en estudios genético cuantitativos. La importancia genética de la selección de toros con base en perímetros escrotales mínimos relacionados con la edad y la raza es respaldada en cuanto a los aspectos fisiológicos, siendo que mayores perímetros escrotales repercuten en mayor producción espermática (Sundararaman y col 2002, Latif y col 2009), mejor calidad seminal (Siddiqui y col 2008, Latif y col 2009), reflejado en mayor eficiencia reproductiva cuando los reproductores son expuestos a la monta natural (Sarder y col 2007, Waldner y col 2010). Sin embargo, otras características testiculares medidas en el examen andrológico, como la consistencia (Coulter y col 1976, Dias y col 2006), volumen (Toelle y Robinson 1985, Ducrocq y Humblot 1995, Dias y col 2006, Boligon y col $2010^{\mathrm{b}}$, Silva y col 2011) y peso de los testículos (Oyeyemi y Eunice 2006) han sido consideradas en estudios genéticos.

Aspectos favorables están asociados a la adopción del PE, siendo fácilmente medido a bajo costo en diferentes edades estandarizadas $\mathrm{y}$, además, genéticamente correlacionado con la libido (Sarreiro y col 2002, Quirino y col 2004), producción espermática (Quirino y col 2004, Kealey y col 2006), calidad seminal (Quirino y col 2004, Kealey y col 2006, Garmyn y col 2011, Silva y col 2011), volumen testicular (Boligon y col 2010 Silva y col 2011), formato testicular (Silva y col 2011), además del peso corporal (Quirino y col 2004, Kealey y col 2006, Barichello y col 2010, Boligon y col 2010a, Yokoo y col 2010) y composición de la carcasa (Yokoo y col 2010).

El PE como criterio de selección de bovinos está indicado en programas de selección teniendo en cuenta que ha obtenido en la mayoría de estudios estimaciones de heredabilidad que fueron de moderada a alta magnitud (Eler y col 2006, Boligon y col 2010 b, Yokoo y col 2010, Silva y col 2011), así como el hecho de estar genéticamente asociado de modo favorable con la fertilidad de hembras (Eler y col 2004, Eler y col 2006, Forni y Albuquerque 2005, Meirelles y col 2009, Van Melis y col 2010a), con características de crecimiento (Quirino y Bergmann 1998, Dias y col 2006, Kealey y col 2006, Yokoo y col 2010) y características indicadoras de la calidad seminal (Martinez y col 2000, Kealey y col 2006, Garmyn y col 2011, Silva y col 2011).

Las correlaciones genéticas entre PE y precocidad sexual y longevidad reproductiva en hembras bovinas, con magnitudes de media a alta, asumen gran importancia, pues indican que la selección de machos con mayores valores de DEP (diferencia esperada en la progenie) para $P E$ revertirá en mayor proporción de hembras precoces sexualmente y longevas en el rebaño (Eler y col 2004, Eler y col 2006, Meirelles y col 2009, Van Melis y col 2010 b). Actualmente, la efectividad de esta selección indirecta se vuelve todavía más consistente por la posibilidad de la evaluación conjunta del PE con la precocidad sexual y longevidad en análisis multicaracterísticos, mejorándose la precisión de predicción del mérito genético de toros jóvenes para estas características (Van Melis y col 2010b).

Por lo tanto, la adopción del PE como criterio de selección permite la búsqueda del mejoramiento de la fertilidad en ambos sexos, con rápido progreso genético en la selección de toros jóvenes buscando aumentar la fertilidad de los rebaños comerciales.

\section{VOLUMEN TESTICULAR (VT)}

El volumen testicular (VT) es una de las características asociadas al desempeño reproductivo de los machos (Bailey y col 1998, Quirino 1999, Unanian y col 2000, Silveira y col 2010). Para algunos autores, el VT sería la medida más adecuada para representar la producción espermática, especialmente en Bos indicus, cuyo formato testicular más alargado repercute en menor diámetro testicular (Bailey y col 1996, Caldas y col 1999, Unanian y col 2000, Viu y col 2006), además de la pared de la bolsa escrotal ser equivocadamente incluida en las medidas (Bailey y col 1998, Viu y col 2006) y según algunos autores no ser predictor de la producción espermática y fertilidad en toros jóvenes (Makarechian y Farid 1985, Coulter y Kozub 1989, Thompson y Jonhson 1995).

A partir del largo y ancho son calculados tanto el volumen como el peso testicular (Fields y col 1979, Toelle y Robinson 1985, Bailey y col 1998). Desde el punto de vista práctico, la obtención de estas medidas para el cálculo del VT es más trabajosa que la medición del PE, sin embargo, son medidas más precisas y podrían ser realizadas en la rutina del examen andrológico.

De acuerdo a las observaciones de Toelle y Robinson (1985), basadas en los estudios de Pelletier y col (1981) y Neely y col (1982), el desarrollo testicular es íntimamente dependiente del crecimiento o ganancia de peso corporal en el período prepúber y, en un segundo momento, se vuelve más dependiente de una compleja interacción de mecanismos hormonales. En este contexto, el VT se ha mostrado como un buen indicador del desarrollo testicular y de la producción espermática en toros jóvenes que alcanzaron la pubertad, condición no evidenciada en machos adultos en relación de la baja correlación entre el peso corporal 
y testicular y la producción de espermatozoides (Willet y Ohms 1957, Bailey y col 1996). Sin embargo, es importante resaltar el efecto ambiental afectando el desarrollo testicular, especialmente el nutricional interfiriendo en el peso corporal (Fields y col 1979, Browning Jr y col 1997, Unanian y col 2000). Se sugirió entonces, que las medidas de biometría testicular deberían ocurrir después de la pubertad, identificando de forma indirecta los individuos de mayor potencial de producción cuantitativa y cualitativa del semen.

Dentro de las medidas biométricas testiculares complementarias al PE, el largo y ancho de los testículos medidos con auxilio de un pie de metro fueron contemplados en estudios genéticos (Toelle y Robinson 1985, Quirino y col 2004, Silveira 2004, Fernandes Júnior 2006) y reproductivos (Bailey y col 1998, Unanian y col 2000, Viu y col 2006, Fernandes Júnior y Franceschini 2007, Silveira y col 2010).

En general, las estimaciones de heredabilidad observadas para las medidas biométricas testiculares y el VT son de magnitud moderada a alta. En la raza Hereford, Toelle y Robinson (1985) estimaron heredabilidades de 0,34 a los 205 días, de 0,21 a los 365 días y de 0,34 para el período de crecimiento (205 a los 365 días). Lunstra y col (1988) obtuvieron estimaciones de heredabilidad de 0,37 en toros jóvenes de diferentes razas europeas (británicas y continentales) (Bos taurus). El mayor valor fue descrito por Ducrocq y Humblot (1995) que encontraron heredabilidad de 0,65 para VT en la raza Normanda. Sin embargo, magnitudes más bajas fueron descritas por Fernandes Júnior (2006), en toros jóvenes híbridos, el cual estimó heredabilidad de 0,22 para la misma característica. Boligon y col $\left(2010^{\mathrm{b}}\right)$ también reportaron magnitudes bajas y moderadas para VT en toros jóvenes de la raza Nelore, siendo de 0,19, 0,26 y 0,39 , para las edades de 9,12 y 18 meses, respectivamente. Considerando 15.313 registros de VT, Silva y col (2011) estimaron heredabilidad de 0,31 , es decir, de moderada magnitud, valor inferior a la heredabilidad obtenida para el PE $\left(h^{2}=0,43\right)$.

Estudiando las correlaciones genéticas incluyendo VT, Dias y col (2006) resaltaron la importancia del desarrollo testicular en la constitución del índice de CAP (Vale Filho y col 1999). Estos autores describieron elevados valores de correlación genética de 0,99 del volumen con el PE y 0,71 del VT con el CAP en toros de la raza Nelore. En la misma raza, Boligon y col $\left(2010^{\mathrm{b}}\right)$ encontraron correlación genética de 0,61 y 0,86 del VT con el PE, en toros jóvenes con 12 y 18 meses de edad. Silva y col (2011) confirmaron la alta correlación genética $(r g=0,94)$ entre PE y VT analizando gran número de registros $(\mathrm{n}=15.313)$. Así, se demostró que ambas características son determinadas en gran parte por los mismos pares de genes.

Toelle y Robinson (1985) obtuvieron correlaciones genéticas favorables con la tasa de preñez y con edad al primer parto en hembras de ganado de carne.
Con base en las heredabilidades obtenidas en los diferentes trabajos, se justifica la continuidad de investigaciones sobre estimativas de parámetros genéticos incluyendo el VT y su correlación con importantes características reproductivas, principalmente con bancos de datos que incluyan gran número de registros, dado que esta característica podrá ser ventajosa como criterio de selección para el aumento de la fertilidad.

Sin embargo, de acuerdo con lo mencionado tanto por Boligon y col $\left(2010^{\mathrm{b}}\right)$ y Silva y col (2011), frente a las ventajas genéticas ya mencionadas para el PE y mayor dificultad en medir el largo y ancho de los testículos, no hay necesidad de adoptarse el VT como criterio alternativo de selección de toros.

\section{FORMATO TESTICULAR}

La caracterización del formato testicular busca la obtención de nuevos conceptos para auxiliar la evaluación de reproductores. Bailey y col (1996) verificaron que toros con menor PE y testículos más alargados podrían tener mayor volumen y/o peso testicular que otros toros dotados de formatos ovoide o esférico y con producción espermática comparable a aquellos de mayor diámetro. Viu y col (2006) concluyeron que, además del PE, el volumen, peso y formato testicular pueden ser parámetros útiles en la selección de reproductores, a pesar de que hayan resaltado el reducido número de estudios correlacionando las variaciones normales del formato testicular a la producción y calidad espermática, especialmente sobre las características del semen de Bos indicus.

Las formas testiculares ovoides son más comunes en Bos taurus al contrario que en Bos indicus donde las alargadas son predominantes (Bailey y col 1996, Unanian y col 2000, Silveira 2004, Fernandes Júnior 2006, Silveira y col 2010, Silva y col 2011), lo que repercute en perímetros escrotales menos expresivos en cebuinos comparado con los taurinos (Unanian y col 2000). Este aspecto fue evidenciado en toros jóvenes híbridos, en que Fernandes Júnior y Franceschini (2007) observaron la predominancia de las formas más esféricas y ovales en individuos con alta proporción de Bos taurus en su composición racial.

Por otro lado, los testículos alargados presentaron mayor superficie de contacto con distribución más uniforme de los vasos sanguíneos y tejido espermático, mayor gradiente de temperatura entre los polos testiculares, menor temperatura media en el microambiente intratesticular, optimización de la termorregulación y, consecuentemente, mejores estándares seminales en condiciones ambientales tropicales (Bailey y col 1996, Lunstra y Coulter 1997, Fernandes Júnior y Franceschini 2007, Silva y col 2011). De esta manera, cuando son expuestos a ambientes de alta temperatura, la disminución en la calidad espermática es menos frecuente e intensa en toros Bos indicus, además de que estos se recuperan más rápidamente, cuando son comparados con toros Bos taurus e híbridos (Brito y col 2004). 
A pesar de que sea potencialmente interesante, es rara la publicación científica con estimaciones de parámetros genéticos del formato testicular en bovinos, como el estudio de Silva y col (2011) sobre parámetros genéticos de las características andrológicas en la especie bovina. Silva y col (2011) mencionaron la necesidad de monitorear el formato testicular en rebaños de razas cebuinas sometidas a selección para PE. Tal preocupación se debe a la correlación genética positiva y alta entre PE y el formato testicular $(r g=0,45)$, indicando que la selección para el PE llevará indirectamente a la obtención de formatos más esféricos, pudiendo a lo largo de las generaciones seleccionadas repercutir en perjuicios en la capacidad termorreguladora del testículo de toros cebuinos. Sin embargo, los autores resaltan que la selección para PE debe continuar y que tal tendencia y posibles consecuencias deberán ser objetivo de futuros estudios en el ámbito reproductivo.

\section{PARÁMETROS GENÉTICOS DE LAS CARACTERÍSTICAS SEMINALES}

El espermograma y la evaluación física del semen proveen informaciones importantes para la determinación de la fertilidad. Correlaciones entre características físicas, como la motilidad progresiva rectilínea, así como la morfología espermática fueron significativas con la fertilidad de toros (Söderquist y col 1996, Peet y col 1998).

Las características seminales son afectadas por diferentes factores ambientales y genéticos, como la edad del animal (Chacón y col 2002, Godfrey y Dodson 2005, Fuerst-Waltl y col 2006), familia (Ducrocq y Humblot 1995), composición racial (Chacón y col 2002, Sylla y col 2007), endogamia en el rebaño (Smith y col 1989, Godfrey y Dodson 2005), estacionalidad (Everett y col 1978, Barth y Waldner 2002) y número de eyaculados y período entre colectas de semen (Mathevon y col 1998, Kapš y col 2000, Chacur y col 2006). Para Higdon III y col (2000), dentro de los factores mencionados, la edad del toro es el componente de mayor impacto en la aptitud reproductiva, dadas las alteraciones fisiológicas que ocurren en detrimento de la madurez sexual y envejecimiento de los animales, consideración también destacada por Ducrocq y Humblot (1998). Por lo tanto, los cuadros espermáticos desfavorables son más incidentes en toros en estado de pubertad (Freneau y col 2006) y viejos (Godfrey y Dodson 2005).

Las asociaciones fenotípicas de las características biométricas testiculares, principalmente el PE, con las características seminales fueron estudiadas por diferentes autores. Godfrey y Dodson (2005) observaron mejor motilidad y menor incidencia de patologías espermáticas con el aumento del PE. En toros de la raza Nelore, Viu y col (2006) relataron correlación negativa entre los defectos espermáticos con el volumen y formato testicular, además del PE.
En contraste con la gran disponibilidad de estudios en las áreas de fisiología y fisiopatología de la reproducción, son pocos los reportes de estimativas de parámetros genéticos para características seminales, principalmente en toros de razas cebuinas. En estos trabajos, las características de producción y calidad del semen fueron contempladas como: concentración espermática, volumen del eyaculado, turbidez, motilidad y vigor espermático y anormalidades de los espermatozoides.

\section{HEREDABILIDAD DE LAS CARACTERÍSTICAS SEMINALES}

En la mayoría de los estudios, las estimaciones de heredabilidad encontradas fueron de baja magnitud, lo que indicó un pequeño componente genético aditivo (Sarreiro y col 2002, Gredler y col 2007, Garmyn y col 2010, Silva y col 2011).

Dentro de las características que integran el examen físico del semen, Kealey y col (2006), en la raza Hereford, estimaron valor moderado de heredabilidad $(0,22)$ para motilidad espermática. Otros estudios presentaron estimaciones de heredabilidad de baja magnitud para motilidad espermática, cuyos valores oscilaron entre 0,01 y 0,16 (Quirino 1999, Sarreiro y col 2002, Dias y col 2006, Garmyn y col 2010, Silva y col 2011). Los reportes de estimación de heredabilidad para vigor espermático y turbidez fueron también discrepantes. Algunos autores describieron baja magnitud para el vigor espermático, de 0,03 a 0,08 (Sarreiro y col 2002, Dias y col 2006, Silva y col 2011). Por lo tanto, se concluye a partir de estas investigaciones que el progreso genético deberá ser lento en caso de que estas características cinéticas de los espermatozoides, como la motilidad y el vigor espermático, sean adoptadas como criterios de selección.

Las estimaciones de heredabilidad reportadas para los defectos espermáticos también oscilaron de baja a moderada magnitud. Previamente Smith y col (1989) ya habían relatado una similar magnitud de heredabilidad, de 0,31 , para defectos espermáticos primarios, pero casi nula para los secundarios, de 0,02 . En un estudio posterior, Kealey y col (2006) encontraron heredabilidad de 0,30 y 0,33 para defectos espermáticos primarios y secundarios, respectivamente. Recientemente, Garmyn y col (2010) reportaron estimaciones de heredabilidad de 0,23, 0,27 y 0,25 para defectos espermáticos primarios, secundarios y totales en machos de la raza Angus. Tales magnitudes de las heredabilidades permitirían, al principio, la adopción del porcentual de defectos espermáticos como criterio de selección para mejoría del perfil seminal y de la fertilidad de toros en rebaños comerciales, estableciéndose un progreso genético más efectivo que para las demás características seminales. Sin embargo, otros autores no corroboran tal suposición, dado que estos encontraron bajas magnitudes de heredabilidad para los defectos espermáticos (Dias y col 2006, Silva 2009). 
Cuando fue considerada la aptitud reproductiva, es decir, la condición del toro ser apto o satisfactorio para la reproducción por la evaluación andrológica, Vale Filho y col (1999) encontraron heredabilidad de elevada magnitud $(0,75)$ para el índice CAP, que los autores colocaron como referencia en la selección de futuros reproductores. De manera diferente, tal vez por la metodología estadística y número de animales empleados, Dias y col (2006) estimaron heredabilidad de baja magnitud para el mismo índice $(0,10)$. Los hallazgos de Dias y col (2006) fueron corroborados por Silva (2009) que también relató una estimativa de heredabilidad de 0,10 , o sea, igualmente baja para la aptitud reproductiva. De esta manera, los estudios más recientes y con mayor número de registros indicaron que el progreso genético deberá ser pequeño y lento si la aptitud andrológica fuera utilizada como criterio de selección.

Otras características seminales, como la concentración espermática y volumen del eyaculado, fueron consideradas en algunos estudios con resultados divergentes. Algunos investigadores reportaron heredabilidad próxima a cero para concentración espermática (Schlote y Munks 1980, Makulska y col 1993, Mathevon y col 1998), mientras que otros como Ducrocq y Humblot (1995) y Kapš y col (2000) estimaron heredabilidad de alta y moderada magnitud, 0,37 y 0,26 , respectivamente. Estas diferencias en magnitud pueden estar relacionadas al número reducido de animales en algunos de los trabajos, así como al método de análisis y modelo estadístico adoptado. En la raza Simmental, Kapš y col (2000) y Gredler y col (2007) reportaron heredabilidad para volumen seminal de baja y moderada magnitud, 0,04 y 0,18 , respectivamente. Ducrocq y Humblot (1995) y Mathevon y col (1998), en razas francesas, estimaron valores elevados de heredabilidad para la misma característica, 0,49 y 0,65 , respectivamente. Estos autores resaltaron la importancia de la inclusión del volumen seminal y concentración espermática en análisis genético debido a la variación entre eyaculados de un mismo toro. En este caso, la definición del método de colecta del semen es un factor importante, pues se espera un aumento del volumen y una disminución de la concentración espermática utilizando el método de electroeyaculación cuando es comparado con el método de vagina artificial.

CORRELACIONES GENÉTICAS INCLUYENDO

CARACTERÍSTICAS SEMINALES

Rege y col (2000) observaron que el porcentaje de anormalidades espermáticas fue negativamente correlacionado con la motilidad y turbidez, además del volumen del semen y concentración espermática, tanto por asociación fenotípica como genética. Silva (2009) encontró correlaciones genéticas favorables entre PE con defectos espermáticos menores $(-0,23)$, defectos espermáticos mayores $(-0,16)$ y defectos espermáticos totales $(-0,25)$, lo que sugiere, aunque las magnitudes no sean altas, que la selección para PE favorecería la disminución de incidencias de patologías espermáticas.

Otras características andrológicas, como la libido y el $\mathrm{PE}$, fueron correlacionadas genéticamente a las seminales. Quirino y col (2004) describieron que toros de mayor libido presentan bajo porcentaje de anormalidades espermáticas, dado que las características morfológicas tuvieron correlación genética favorable con la libido. Los autores también encontraron asociación genética alta y negativa entre el volumen seminal y motilidad espermática con la libido.

Correlaciones genéticas favorables entre el PE y características de motilidad y morfología espermática fueron estimadas en distintos trabajos. Las magnitudes de las correlaciones genéticas entre PE y motilidad espermática fueron de moderada $(0,34)$, como la obtenida por Kealey y col (2006), a alta $(0,72)$ como la obtenida por Dias y col (2006). Entre el PE y defectos espermáticos, las magnitudes también oscilaron, de $-0,12$ a $-0,67$, por tanto, de baja a alta magnitud (Dias y col 2006, Kealey y col 2006). Vale Filho y col (1999), en toros de la raza Nelore, encontraron correlaciones genéticas favorables entre el índice de clasificación andrológica (CAP) con motilidad, vigor y turbidez, al igual que con defectos espermáticos y peso corporal.

Las características seminales no figuran como potenciales criterios de selección, de acuerdo con los bajos valores de heredabilidades reportados en la mayoría de los artículos disponibles. Sin embargo, interesantes correlaciones genéticas fueron favorables con $\mathrm{PE}$, indicando que la selección para PE tenderá a repercutir en una mejor calidad seminal, a pesar de que tal respuesta indireta sea lenta y de baja magnitud.

\section{PARÁMETROS GENÉTICOS PARA LA LIBIDO}

Además de las características testiculares y seminales, la habilidad del toro para identificar y copular con la hembra en estro, es decir, la libido, también es importante para establecerse el potencial reproductivo del macho, a pesar de que ocurran diferencias respecto a las metodologías en la aplicación de la prueba, especialmente en toros cebuinos.

La mayor contribución práctica en la identificación de toros de alta libido en el rebaño es la posibilidad de alteración a la proporción tradicional toro:vaca de 1:25 para 1:60, 1:80 y hasta 1:100 (Fonseca y col 2000, Santos y col 2003, Santos y col 2004, Franco y col 2006), repercutiendo en reducciones en el costo de cada becerro destetado.

Sin embargo, la metodología del test de la libido en la evaluación del comportamiento sexual de toros y del real potencial reproductivo de la especie en condiciones extensivas de cría es cuestionada (Santos y col 2004, Oliveira y col 2007, Dias y col 2009, Scheepers y col 2010), especialmente cuando es empleada en toros cebuinos (Galina y col 2007, Katz y col 2008). Algunas inconsistencias del test son reportadas (Petherick 2005), como: tiempo 
de realización de los test (Santos y col 2004); período del día en que el test fue realizado (Oliveira y col 2007), y respuesta de los animales (Dias y col 2009). Algunos autores observaron correlaciones lineales bajas o nulas entre la libido y la calidad espermática, libido y circunferencia escrotal y entre la libido y la testosterona (Santos y col 2003, Santos y col 2004, Chacur y col 2007, Scheepers y col 2010), lo que sugiere al test de la libido apenas como evaluación complementaria al examen andrológico.

De acuerdo con algunos autores, la libido es influenciada por factores genéticos (Ologun y col 1981, Bench y col 2001, Quirino y col 2004). Estudios realizados en toros de la raza Nelore estimaron heredabilidades para la libido con moderada magnitud, 0,19 (Quirino y col 2004) y 0,31 (Sarreiro y col 2002), teniendo el peso corporal como covariable en el modelo matemático y 0,33 (Sarreiro y col 2002) y 0,34 (Quirino y col 2004) excluyendo el peso corporal del modelo. De esta forma, se espera que la selección directa para la libido pueda resultar en el rápido progreso genético.

Diferentes autores demostraron que existen asociaciones fenotípicas favorables entre la libido y otras características andrológicas, como el PE, motilidad y defectos espermáticos (Smith y col 1981, Chenoweth 1983, Quirino y col 2004). Sin embargo, estas correlaciones fueron bajas y con probabilidad de ser influenciadas por la edad del toro como fue encontrado en otros estudios (Hoflack y col 2003, Santos y col 2004). A nivel genético, Sarreiro y col (2002) observaron que la libido está asociada favorablemente a las características seminales y al PE, indicando que la selección para la libido puede llevar a la selección indirecta para estas características, con excepción del vigor espermático. No obstante, tales condiciones no fueron evidenciadas por Quirino y col (2004), ya que las correlaciones genéticas entre la libido y los defectos espermáticos y con el PE se mostraron desfavorables. Pese a esto, Quirino y col (2004) demostraron la existencia de correlación genética alta y favorable entre la libido y el peso corporal, sugiriendo que la selección para una de esas características puede llevar a la selección indirecta para la otra.

La libido del toro está influenciada por diversos factores ambientales y también genéticos; sin embargo, para indicar esta característica como criterio de selección es necesaria la realización de estudios adicionales en el ámbito reproductivo y genético, dado que los criterios en la adopción del test en campo todavía son controvertidos especialmente en toros cebuinos criados en condiciones extensivas.

\section{CONCLUSIONES}

El conocimiento de las heredabilidades y las correlaciones genéticas de las características testiculares y seminales, además del comportamiento sexual, provenientes de bancos de datos confiables, podrán proveer alternativas para la elaboración de estrategias más adecuadas de selección para fertilidad.

El examen andrológico y las pruebas de comportamiento sexual determinan el potencial reproductivo de toros jóvenes, permitiendo establecer prácticas de manejo y proporción de toro y hembras optimizadas, y por medio de la estimación de parámetros genéticos de las características andrológicas nuevos criterios de selección podrán ser adoptados buscando el aumento de la fertilidad de toros destinados a la reproducción en rebaños comerciales.

\section{RESUMEN}

La precocidad sexual y fertilidad tienen gran impacto en el éxito económico de los rebaños bovinos comerciales, en que las características reproductivas han sido adoptadas como criterios de selección. Sin embargo, la mayoría de estas características dependen de eventos reproductivos de las hembras y, con excepción del perímetro escrotal, pocos estudios abordan las características andrológicas. Las estimaciones de las heredabilidades y correlaciones genéticas de las características testiculares y seminales, además del comportamiento sexual, podrán proveer alternativas para la elaboración de estrategias más adecuadas de selección para fertilidad, en conjunto con las demás características de interés económico.

\section{REFERENCIAS}

Abadia D, JS Brinks, EJ Carroll. 1976. Genetics of seminal traits in young beef bulls. J Anim Sci 42, 1552.

Alexander JH. 2008. Bull breeding soundness evaluation: A practitioner's perspective. Theriogenology 70, 469-472.

ANUALPEC. 2010. Anuário da Pecuária Brasileira. Instituto Agra FNP, São Paulo, Brasil.

Bailey TL, DR Monke, RS Hudson, DF Wolfe, RL Carson, G Riddell. 1996. Testicular shape and its relationship to sperm production in mature Holstein bulls. Theriogenology 46, 881-887.

Bailey TL, TA Hudson, TA Powe, MG Riddell, DF Wolfe, RL Carson. 1998. Caliper and ultrasonographic measurements of bovine testicles and a mathematical formula for determining testicular volume and weight in vivo. Theriogenology 49, 581-594.

Barichello F, MM Alencar, RAA Torres Junior, LO Silva. 2010. Heritability and correlations for weight, scrotal circumference and visual scores at weaning, in Canchim beef cattle. Pesq Agropec Bras 45, 563-570.

Barth AD, CL Waldner. 2002. Factors affecting breeding soundness classification of bulls examined at the Western College of Veterinary Medicine. Can Vet J 43, 274-284.

Bench CJ, EO Price, MR Dally, R Borgwardt. 2001. Artificial selection of rams for sexual performance and its effect on the sexual behavior and fecundity of male and female progeny. Appl Anim Behav Sci 72, 41-50.

Boligon AA, LG Albuquerque, MEZ Mercadante, RB Lôbo, JA II V Silva, RC Sesana, JC Sesana, JB Junqueira. 2010 ${ }^{\text {. Study of relations }}$ among age at first calving, average weight gains and weights from weaning to maturity in Nellore cattle. Rev Bras Zootec 39, 746-751.

Boligon AA, JA II V Silva, RC Sesana, JC Sesana, JB Junqueira, LG Albuquerque. $2010^{\mathrm{b}}$. Estimation of genetic parameters for body weights, scrotal circumference, and testicular volume measured at different ages in Nellore cattle. J Anim Sci 88, 1215-1219.

Brito LFC, AEDF Silva, RT Barbosa, JP Kastelic. 2004. Testicular thermoregulation in Bos indicus, crossbred and Bos taurus bulls: relationship with scrotal, testicular vascular cone and testicular morphology, and effects on semen quality and sperm production. Theriogenology 61, 511-528.

Browning Jr R, BG Warrington, JW Holloway, RD Randel. 1997. Testicular size at weaning in tropically-adapted beef bulls as influenced by breed of sire and dam. Theriogenology 48, 257-265. 
Brun JM, M Theau-Clement, G Bolet. 2002. Evidence for heterosis and maternal effects on rabbit semen characteristics. Anim Res 51, 433-442.

Caldas ME, TG Pinho, PA Pinto, LAG Nogueira. 1999. Avaliação da biometria e morfologia testicular de touros jovens da raça Nelore (Bos taurus indicus). Rev Bras Reprod Anim 23, 210-212.

Chacón J, E Pérez, H Rodríguez-Martínez. 2002. Seasonal variations in testicular consistency, scrotal circumference and spermiogramme parameters of extensively reared Brahman (Bos indicus) bulls in the tropics. Theriogenology 58, 41-50.

Chacur MGM, MC Araújo, SN Kronka. 2006. Características seminais, corpóreas e anatômicas do aparelho reprodutor de reprodutores da raça Canchim aos 14 e 48 meses de idade. Arq Cien Vet Zool UNIPAR 9, 21-27.

Chacur MGM, FP Sirchia, EP Zerbinatti, SN Kronka, E Oba. 2007. Relação entre circunferência escrotal, libido, hormônios e características do sêmen em touros Brangus e Pardo-Suíço. Acta Sci Vet 35, 173-179.

Chandler JE, RW Adkinson, GM Hay, RL Crain. 1985. Environmental and genetic sources of variation for seminal quality in mature Holstein bulls. J Dairy Sci 68, 1270-1279.

Chenoweth PJ. 1983. Sexual behavior of the bull: a review. J Dairy Sci 66, 173-179.

Chenoweth PJ, FM Hopkins, JC Spitzer, RE Larsen. 1993. Guidelines for using the bull breeding evaluation form. In: Chenoweth PJ (ed). Theriogenology Handbook. $1^{\text {st }}$ ed. Society for Theriogenology, Montgomery, USA, Pp B-10.

Coulter GH, TR Rounsaville, RH Foote. 1976. Heritability of testicular size and consistency in Holstein bulls. J Dairy Sci 43, 9-11.

Coulter GH, GC Kozub. 1989. Efficacy of methods used to test fertility of beef bulls used for multiple-sire breeding under range conditions. J Anim Sci 67, 1757-1766.

David I, X Druart, G Lagriffoul, E Manfredi, C Robert-Granié, L Bodin. 2007. Genetic and environmental effects on semen traits in Lacaune and Menech tête rousse AI rams. Genet Sel Evol 39, 405-419.

Dias JC, VJ Andrade, AB Fridrich, DF Salvador, VR Vale Filho, AB Corrêa, MA Silva. 2006. Estimativas de parâmetros genéticos de características reprodutivas de touros Nelore, de dois e três anos de idade. Arq Bras Med Vet Zootec 58, 388-393.

Dias JC, VJ Andrade, LL Emerick, JAM Martins, VR Vale Filho, MA Silva. 2009. Teste da libido em touros jovens Guzerá e suas associações com características reprodutivas e níveis séricos de testosterona. Arch Vet Sci 14, 204-213.

Ducrocq V, P Humblot. 1995. Genetic characteristics and evolution of semen production of young Normande bulls. Livest Prod Sci 41, 1-10.

Ducrocq V, P Humblot. 1998. Is selection on semen characteristics needed? Interbull Bulletin 18, 68-73.

Eler JP, JAIIV Silva, JL Evans, JBS Ferraz, F Dias, BL Golden. 2004. Additive genetic relationships between heifer pregnancy and scrotal circumference in Nellore cattle. J Anim Sci 82, 2519-2527.

Eler JP, JBS Ferraz, JCC Balieiro, EC Mattos, GB Mourão. 2006. Genetic correlation between heifer pregnancy and scrotal circumference measured at 15 and 18 months of age in Nellore cattle. Genet Mol Res 5, 565-570.

England GCW, L Phillips, SL Freeman. 2010. Heritability of semen characteristics in dogs. Theriogenology 74, 1136-1140.

Everett RW, B Bean, RH Foote. 1978. Sources of variation of semen output. J Dairy Sci 61, 90-95.

Euclides Filho K. 1999. Herdabilidade. In: Euclides Filho K (ed). Melhoramento Genético Animal no Brasil: Fundamentos, História e Importância. $1^{\text {st }}$ ed. Embrapa Gado de Corte, Campo Grande, Brasil, Pp 1-13.

Falconer DS, T Mackay. 1996. Introduction to quantitative genetics. $4^{\text {th }}$ ed. Longman Group Limited, London, UK.

Fernandes Júnior JA. 2006. Estimativas de parâmetros genéticos para perímetro escrotal, volume testicular e aptidão reprodutiva e de biometria testicular em uma população de bovinos compostos. Tesis Doctoral, Faculdad de Ciencias Agrárias y Veterinárias, Universidad Estadual Paulista, Jaboticabal, São Paulo, Brasil.
Fernandes Júnior JA, PA Franceschini. 2007. Maturidade sexual e biometria testicular de touros jovens compostos Montana Tropical ${ }^{\circledR}$ criados a pasto. Ars Vet 23, 59-66.

Ferraz JBS, JP Eler, BL Golden. 1999. A formação do composto Montana Tropical. Rev Bras Reprod Anim 23, 115-117.

Fields MJ, WE Burns, AC Warnick. 1979. Age, season and breed effects on testicular volume and semen traits in young beef bulls. J Anim Sci 48, 1299-1304.

Folhadella IM, WF Sá, AM Ferreira, LSA Camargo, JHM Viana, AA Ramos, MVGB Silva. 2006. Características andrológicas de touros da raça Gir. Arq Bras Med Vet Zootec 58, 809-815.

Fonseca VO, JAG Bergmann, CS Franco. 1997. Potencial reprodutivo de touros da raça Nelore (Bos taurus indicus) acasalados com elevado número de vacas. Arq Bras Med Vet Zootec 49, 53-62.

Fonseca VO, CS Franco, JAG Bergmann. 2000. Potencial reprodutivo e econômico de touros Nelore acasalados coletivamente na proporção de um touro para 80 vacas. Arq Bras Med Vet Zootec 52, 77-82.

Forni S, LG Albuquerque. 2005. Estimates of genetic correlations between days to calving and reproductive and weight traits in Nelore cattle. J Anim Sci 83, 1511-1515.

Franco CS, VO Fonseca, L Gaste. 2006. Potencial reprodutivo de touros Nelore acasalados coletivamente na proporção de um touro para 100 vacas. Arq Bras Med Vet Zootec 58, 1156-1161.

Freneau GE, VR Vale Filho, AP Marques Jr, WS Maria. 2006. Puberdade em touros Nelore criados em pasto no Brasil: características corporais, testiculares e seminais e de índice de capacidade andrológica por pontos. Arq Bras Med Vet Zootec 58, 1107-1115.

Fuerst-Waltl B, H Schwarzenbacher, C Perner, J Sölkner. 2006. Effect of age and environmental factors of semen production and semen quality of Austrian Simmental bulls. Anim Reprod Sci 95, 27-37.

Furstoss V, I David, B Leboeuf, P Guillouet, P Boué, L Bodin. 2009. Genetic and non-genetic parameters of several characteristics of production and semen quality in young bucks. Anim Reprod Sci 110, 25-36.

Galina CS, MM Horn, R Molina. 2007. Reproductive behavior in bulls raised under tropical and subtropical conditions. Hormones Behav 52, 26-31.

García-Tomáz M, J Sánchez, O Rafel, J Ramon, M Piles. 2006. Variability, repeatability and phenotypic relationships of several characteristics of production and semen quality in rabbit. Anim Reprod Sci 93, 88-100.

Garmyn AJ, DW Moser, RA Christmas, J Minick Bormann. 2011. Estimation of genetic parameters and effects of cytoplasmic line on scrotal circumference and semen quality traits in Angus bulls. J Anim Sci 89, 693-698.

Godfrey RW, RE Dodson. 2005. Breeding soundness evaluations of Senepol bulls in US Virgin Islands. Theriogenology 63, 831-840.

Graffer T, H Solbu, O Filseth. 1988. Semen production in artificial insemination bulls in Norway. Theriogenology 30, 1011-1121.

Gredler B, C Fuerst, B Fuerst-Waltl, H Schwarzenbacher, J Sölkner. 2007. Genetic parameters for semen production traits in Austrian dual-purpose simmental bulls. Reprod Domest Anim 42, 326-328.

Henry M, JP Neves. 1998. Parâmetros de avaliação andrológica por espécie e padrões para julgamento de sêmen de doadores. En: Henry M, JP Neves (ed). Manual para exame andrológico e avaliação de sêmen animal. $2^{\text {da }}$ ed. Colégio Brasileiro de Reprodução Animal, Belo Horizontal, Brazil, Pp 19-46.

Higdon III HL, JC Spitzer, FM Hopkins, WC Bridges Jr. 2000. Outcomes of breeding soundness evaluation of 2898 yearling bulls subjected to different classification systems. Theriogenology 53, 1321-1332.

Hoflack G, A Van Soom, D Maes, A de Kruif, G Opsomer, L Duchateau. 2003. Breeding soundness and libido of Belgian Blue and Holstein Frisian artificial insemination bulls in Belgium and Netherlands. Theriogenology 66, 207-216.

IBGE, Instituto Brasileiro de Geografia e Estatística. 2009. Produção da Pecuária Municipal. Prod Pec Munic IBGE No 37. 
Kabir M, OO Oni, GN Akpa1. 2007. Osborne selection index and semen traits interrelationships in Rhode Island Red and White Breeder cocks. Int J Poult Sci 6, 999-1002.

Kapš M, M Posavi, N Stipić, B Mikulić. 2000. Genetic evaluation of semen and growth traits of young simmental bulls in performance test. Agric Conspec Sci 65, 15-20.

Katz LS. 2008. Variation in male sexual behavior. Anim Reprod Sci $105,64-71$

Kealey CG, MD Macneil, MW Tess, TW Geary, RA Bellows. 2006. Genetic parameter estimates for scrotal circumference and semen characteristics of Line 1 Hereford bulls. J Anim Sci 84, 283-290.

Knights SA, RL Baker, D Gianola, JB Gibb. 1984. Estimates of heritabilities and genetic and phenotypic correlations among growth and reproductive traits in yearling Angus bulls. J Anim Sci 58, 887-893.

Lavara R, ML García, C Torres, JS Vicente, M Baselga. 2008ª Genetic parameters for semen traits of rabbit males: I. Production, morphology, and sperm head morphometry. Proceedings of the 9th WRSA Congress, Verona, Italy, Pp 153-158.

Lavara R, ML García, C Torres, JS Vicente, M Baselga. 2008 ${ }^{\text {. Genetic }}$ parameters for semen traits of rabbit males: II. Motility. Proceedings of the 9th WRSA Congress, Verona, Italy, Pp 159-162.

Latif MA, JU Ahmed, MMU Bhuiyan, M Shamsuddin. 2009. Relationship between scrotal circumference and semen parameters in crossbred bulls. Bangl Vet 26, 61-67.

Lunstra DD, GH Coulter. 1997. Relationship between scrotal infrared temperature patterns and natural-mating fertility in beef bulls. J Anim Sci 75, 767-774.

Lunstra DD, KE Gregory, LV Cundiff. 1988. Heritability estimates and adjustments factors for the effects of bull age and age of dam on yearling testicular size in different breeds of bulls. Theriogenology 30, 127-136.

Makarechian M, A Farid. 1985. The relationship between breeding soundness evaluation and fertility of beef bulls under group mating at pasture. Theriogenology 23, 887-898.

Makulska J, C Hagger, N Kunzi, HU Kupferschmied. 1993. Genetic and environmental influences on semen traits in A.I. bulls. Reprod Domest Anim 28, 279-284.

Martinez ML, RS Verneque, RL Teodoro, LRO Paula, M Cruz, JP Campos, LH Rodrigues, J Oliveira, F Vieira, JH Bruschi, MC Durães. 2000. Correlações entre características da qualidade do sêmen e a circunferência escrotal de reprodutores da raça Gir. Rev Bras Zootec 29, 700-706.

Mathevon M, JCM Dekkers, MM Buhr. 1998. Environmental, management and genetic factors affecting semen production in French Montbéliard bulls. Livest Prod Sci 55, 65-77.

Meirelles SL, AC Espasandin, M Mattar, SA Queiroz. 2009. Genetic and environmental effects on sexual precocity traits in Nellore cattle. $R$ Bras Zootec 38, 1488-1493.

Neely JD, BH Johnson, EU Dillard, OW Robison. 1982. Genetic parameters for testes size and sperm number in Hereford bulls. J Anim Sci 55, 1033-1040.

Oh SH, MT See, TE Long, JM Galvin. 2003. Genetic correlations between boar semen traits. J Anim Sci 81, 317.

Oh SH, MT See, TE Long, JM Galvin. 2006. Genetic parameters for various random regression models to describe total sperm cells per ejaculate over the reproductive lifetime of boars. J Anim Sci $84,538-545$.

Oliveira CB, JD Guimarães, EP Costa, JB Siqueira, CAA Torres, GR Carvalho, SEF Guimarães. 2007. Avaliação do comportamento sexual em touros Nelore: comparação entre os testes da libido em curral e do comportamento sexual a campo. Rev Bras Zootec 36, 32-42.

Ologun AGP, JS Chenoweth, JS Brinks. 1981. Relationships among production traits and estimates of sex-drive and dominance value in yearling beef bulls. Theriogenology 15, 379-788.

Oyeyemi MO, TB Eunice. 2006. Testicular parameters and morphological characteristics of testicular and epididymal spermatozoa of white Fulani bulls in Nigeria. Int J Morphol 24, 175-180.
Peet RL, P Kluck, M Mccarth. 1998. Infertility in Murray Gray bulls associated with abaxial and swollen midpiece sperm defects. Aust Vet J 65, 359-360.

Pelletier J, S Carrez-Camous, JC Thiery. 1981. Basic neuroendocrine events before puberty in cattle, sheep and pigs. J Reprod Fertil 30, 91.

Petherick JC. 2005. A review of some factors affecting the expression of libido in beef cattle, and individual bull and herd fertility. Appl Anim Behav Sci 90, 185-205.

Quirino CR, JAG Bergmann. 1998. Heritability of scrotal circumference adjusted and unadjusted for body weight in Nellore bulls, using univariate and bivariate animal models. Theriogenology 49, 1389-1396.

Quirino CR. 1999. Herdabilidades e correlações genéticas entre medições testiculares, características seminais e libido em touros Nelore. Tesis Doctoral, Escuela de Veterinária, Universidad Federal de Minas Gerais, Belo Horizonte, Minas Gerais, Brasil.

Quirino CR, JAG Bergmann, VR Vale Filho, VJ Andrade, SR Reis, RM Mendonça, CG Fonseca. 2004. Genetic parameters of libido in Brazilian Nellore bulls. Theriogenology 62, 1-7.

Rege JEO, F Toe, E Mukasa-Mugerwa, S Tembely, D Anindo, RL Baker, A Lahlou-Kassi. 2000. Reproductive characteristics of Ethiopian highland sheep II. Genetic parameters of semen characteristics and their relationships with testicular measurements in ram lambs. Small Ruminant Res 37, 173-187.

Robinson J, M Buhr. 2005. Impact of genetic selection on management of boar replacement. Theriogenology, 63, 668-678.

Santos MD, CAA Torres, JD Guimarães, JRM Ruas, GR Carvalho. 2003. Libido de touros Nelore: efeito da proporção touro: vaca sobre a taxa de gestação. Arq Bras Med Vet Zootec 55, 293-300.

Santos MD, CAA Torres, JRM Ruas, JD Guimarães, JM Silva Filho, EP Costa, JC Pereira. 2004. Teste da libido e atividade de monta em touros da raça Nelore. Arq Bras Med Vet Zootec 56, 504-510.

Sarder MJU, MSR Sultana, L Nahar, MA Islam. 2007. Phenotypic characteristics of individual bull used for artificial insemination (AI) programme in Bangladesh. J bio-sci 15, 99-109.

Sarreiro LC, JAG Bergmann, CR Quirino, NR Pineda, VCP Ferreira, MA Silva. 2002. Herdabilidade e correlação genética entre perímetro escrotal, libido e características seminais de touros Nelore. Arq Bras Med Vet Zootec 54, 602-608.

Scheepers SM, CH Annandale, EC Webb. 2010. Relationship between production characteristics and breeding potential of 25-month old extensively managed Bonsmara bulls. S Afri J Anim Sci 40, 163-172.

Schlote W, J Munks. 1980. Population parameters of semen characteristics and non return rates of test bulls. Proceedings of the 31th JEVT Congress, Munich, Germany.

Siddiqui AR, J Bhattacharjee, ZC Das, MM Islam, MA Islam, MA Haque, JJ Parrish, M Shamsuddin. 2008. Crossbred bull selection for bigger scrotum and shorter age at puberty with potentials for better quality semen. Reprod Domest Anim 43, 74-79.

Silva, MR. 2009. Estudo genético quantitativo das características andrológicas de touros jovens da raça Nelore. Tesis Doctoral, Faculdad de Ciencias Agrárias y Veterinárias, Universidad Estadual Paulista, Jaboticabal, São Paulo, Brasil.

Silva MR, VB Pedrosa, JCB Silva, JP Eler, JD Guimarães, LG Albuquerque. 2011. Testicular traits as selection criteria for young Nellore bulls. J Anim Sci 89, 2061-2067.

Silveira TS. 2004. Estádio de maturidade sexual e estimativas de parâmetros genéticos e fenotípicos de características reprodutivas e ponderais, em touros da raça Nelore. Memoria de título, Departamento de Medicina Veterinária, Universidad Federal de Viçosa, Viçosa, Minas Gerais, Brasil.

Silveira TS, JB Siqueira, SEF Guimarães, TAR Paula, TM Neto, JD Guimarães. 2010. Maturação sexual e parâmetros reprodutivos em touros da raça Nelore criados em sistema extensivo. $R$ Bras Zootec 39, 503-511.

Smital J, J Wolf, LL De Sousa. 2005. Estimation of genetic parameters of semen characteristics and reproductive traits in AI boars. Anim Reprod Sci 86, 119-130. 
Smith MF, DL Morris, MS Amoss, NR Parish, JD Williams, JN Wiltbank. 1981. Relationships among fertility, scrotal circumference, seminal quality, and libido in Santa Gertrudis bulls. Theriogenology 6, 379-397.

Smith BA, JG Brinks, GV Richardson. 1989. Estimation of genetic parameters among breeding soundness examination components and growth traits in yearling bulls. J Anim Sci 67, 2892-2896.

Snowder GD, JN Stellflug, LD Van Vleck. 2004. Genetic correlation of ram sexual performance with ewe reproductive traits of four sheep breeds. Appl Anim Behav Sci 88, 253-261.

Söderquist L, Janson L, M Haard, S Einarsson. 1996. Influence of season, age, breed and some others factor on the variation in sperm morphological abnormalities in Swedish dairy AI bulls. Anim Reprod Sci 44, 91-98.

Stalhåmmar EM, L Janson, J Philipsson. 1989. Genetic studies on fertility in AI bulls. I. Age, season and genetic effects on semen characteristics in young bulls. Anim Reprod Sci 19, 1-17.

Sundararaman MN, P Thangaraju, MJ Edwin. 2002. Age related changes in testes size of Jersey bulls and its effects on semen production traits. Indian J Anim Sci 72, 567-568.

Sylla L, G Stradaioli, S Borgami, M Monaci. 2007. Breeding soundness examination of Chianina, Marchigiana, and Romagnola yearling bulls in performance tests over a 10-year period. Theriogenology 67, 1351-1358.

Taylor JF, B Bean, CE Marshall, JJ Sullivan. 1985. Genetic an environmental components of semen production traits of artificial insemination Holstein bulls. J Dairy Sci 68, 2703-2722.

Thompson JA, WH Johnson. 1995. Scrotal size of yearling sires and early calving in beef herds: Epidemiological investigation of possible causal pathways. Theriogenology 43, 1279-1287.

Toelle VD, OW Robinson. 1985. Estimates of genetic correlations between testicular measurements and female reproductive traits in cattle. J Anim Sci 60, 89-100.

Unanian MM, AFDF Silva, C McManus, EP Cardoso. 2000. Características Biométricas Testiculares para Avaliação de Touros Zebuínos da Raça Nelore. Rev Bras Zootec 29, 136-144.

Vale Filho VR. 1989. Padrões do sêmen bovino para o Brasil: Análise e sugestões. Actas VII Congreso Brasileiro de Reproducción Animal, Belo Horizonte, Brasil, Pp 94-118.
Vale Filho VR, CR Quirino, VJ Andrade, JAG Bergmann, SR Reis, RMA Mendonça, CG Fonseca. 1999. Parâmetros genéticos da classificação andrológica por pontos (CAP), em touros da raça Nelore. Rev Bras Reprod Anim 23, 253-255.

Van Eldik P, EH Van Der Waaij, B Ducro, AW Kooper, TAE Stoot, B Colenbrander. 2006. Possible negative effects of inbreeding on semen quality in Shetland pony stallions. Theriogenology 65, 1159-1170.

Van Melis MH, HN Oliveira, JP Eler, JBS Ferraz, J Casellas, L Varona. $2010^{\mathrm{a}}$. Additive genetic relationship of longevity with fertility and production traits in Nellore cattle based on bivariate models. Genet Mol Res 9, 176-187.

Van Melis MH, JP Eler, GJM Rosa, JBS Ferraz, LGG Figueiredo, EC Mattos, HN Oliveira. 2010 ${ }^{\mathrm{b}}$. Additive genetic relationships between scrotal circumference, heifer pregnancy and stayability in Nellore cattle. J Anim Sci, 88, 3809-3813

Viu MAO, CU Magnabosco, HT Ferraz, ML Gambarini, BD Oliveira Filho, DT Lopes, AMF Viu. 2006. Desenvolvimento ponderal, biometria testicular e qualidade seminal de touros Nelore (Bos taurus indicus) criados extensivamente na região centro-oeste do Brasil. Arch Vet Sci 11, 53-57.

Waldner CL, RI Kennedy, CW Palmer. 2010. A description of the findings from bull breeding soundness evaluations and their association with pregnancy outcomes in a study of western Canadian beef herds. Theriogenology 74, 871-883.

Willet EL, JI Ohms. 1957. Measurements of testicular size and its relationship to production of spermatozoa by bulls. J Dairy Sci 40, 1559-1569.

Wolf J. 2009. Genetic parameters for semen traits in AI boars estimated from data on individual ejaculates. Reprod Domest Anim 44, 338-344.

Wolf J. 2010. Heritabilities and genetic correlations for litter size and semen traits in Czech Large White and Landrace pigs. J Anim Sci 88, 2893-2903.

Yokoo MJ, RB Lôbo, FRC Araujo, LAF Bezerra, RD Sainz, LG Albuquerque. 2010. Genetic associations between carcass traits measured by real-time ultrasound and scrotal circumference and growth traits in Nelore cattle. J Anim Sci 88, 52-58. 